\title{
On a Caputo conformable inclusion problem with mixed Riemann-Liouville conformable integro-derivative conditions
}

\author{
Dumitru Baleanu ${ }^{1,2}$, Sina Etemad ${ }^{3}$ and Shahram Rezapour ${ }^{4,5,6^{*}}$ (D)
}

\section{"Correspondence:}

shahramrezapour@duytan.edu.vn;

sh.rezapour@mail.cmuh.org.tw;

sh.rezapour@azaruniv.ac.ir;

rezapourshahram@yahoo.ca

${ }^{4}$ Institute of Research and

Development, Duy Tan University, Da Nang 550000, Vietnam

${ }^{5}$ Faculty of Natural Sciences, Duy Tan University, Da Nang 550000,

Vietnam

Full list of author information is available at the end of the article

\begin{abstract}
We discuss some existence criteria for a new category of the Caputo conformable differential inclusion furnished with four-point mixed Riemann-Liouville conformable integro-derivative boundary conditions. In this way, we employ some analytical techniques on $\alpha-\psi$-contractive mappings and operators having the approximate endpoint property to reach desired theoretical results. Finally, we provide an example to illustrate our last main result.
\end{abstract}

MSC: Primary 34A08; secondary 34A12

Keywords: $\alpha-\psi$-contractive mappings; Approximate endpoint property; Mixed integro-derivative conditions; The Caputo conformable derivative

\section{Introduction}

From a long time ago, human beings have been thinking about finding the secrets and phenomena of the world around in order to be able to answer some questions. For this reason, by increasing its knowledge, the mankind invoked new logical and computational tools. The mathematical operators are one of these useful tools for modeling natural processes in the world. Over the years, mathematicians have introduced various operators for different models, but since fractional order modelings are more accurate than integer order ones, new fractional operators have been defined for this purpose today. In the meantime, the Caputo and the Riemann-Liouville fractional operators have been used more than other operators for complicated fractional modelings (see, for example, [1-5]). Lately, the Hadamard and Caputo-Hadamard fractional operators have been introduced by some researchers and then different modelings have been studied using these operators (see, for instance, [6-9]). In 2015, Caputo and Fabrizio [10] presented a new fractional derivative without singular kernel entitled fractional Caputo-Fabrizio operator, and in the same year, Losada and Nieto [11] investigated some properties of this new fractional operator. Some flexible properties of this nonsingular operator led to numerous papers on the various fractional modelings in this regard (see, for example, [12-14]).

Following this path, Abdeljawad [15] developed the concepts introduced in [16] and investigated some properties of the well-behaved conformable fractional derivatives. In a

(c) The Author(s) 2020. This article is licensed under a Creative Commons Attribution 4.0 International License which permits use sharing, adaptation, distribution and reproduction in any medium or format, as long as you give appropriate credit to the original author(s) and the source, provide a link to the Creative Commons licence, and indicate if changes were made. The images or other third party material in this article are included in the article's Creative Commons licence, unless indicated otherwise in a credit line to the material. If material is not included in the article's Creative Commons licence and your intended use is not permitted by statutory regulation or exceeds the permitted use, you will need to obtain permission directly from the copyright holder. To view a copy of this licence, visit http://creativecommons.org/licenses/by/4.0/. 
paper published in 2017, Jarad et al. [17] wanted to answer the question if one can extend the standard Riemann-Liouville integral of fractional order so that we obtain a unification to other fractional operators including Riemann-Liouville, Caputo, Hadamard, CaputoHadamard, and other derivatives [18]. To reach this goal, the authors introduced novel integration and differentiation operators of fractional order based on conformable operators. Indeed, they defined new functional spaces and established some basic properties of these new combined operators. After that manuscript, a limited number of papers, which rely on these novel operators, have been published so far. For instance, in the following paper for the first time the authors applied new Caputo and Riemann-Liouville conformable operators in their BVP. In fact, Aphithana et al. [19] sketched a new problem as the conformable differential equation of Caputo type with four-point integral conditions

$$
\begin{cases}{ }^{{ }^{C}} \mathcal{D}_{a}^{v, \varrho} w(s)=\breve{g}(s, w(s)) & (a<s<T), \\ w(a)=\mu_{1} w(\xi)+\mu_{2}, & w(T)=\lambda^{\mathrm{RC}} \mathcal{I}_{a}^{v, \beta} w(\sigma),\end{cases}
$$

where ${ }^{\mathrm{CC}} \mathcal{D}_{a}^{v, \varrho}$ is the conformable derivative of Caputo type of order $\varrho \in(1,2]$ with $v \in$ $(0,1]$. Also, ${ }^{\mathrm{RC}} \mathcal{I}_{a}^{v, \beta}$ is a conformable integral of Riemann-Liouville type of order $\beta>0$. They employed some functional analysis techniques to obtain their desired results. Further, different kinds of Ulam stability of the solutions are investigated by them [19]. Also, one can find different applied type works, where researchers use fractional models [20-32].

By utilizing these new operators introduced in [17] and motivated by the abovementioned work, we designed the following Caputo fractional conformable differential inclusion:

$$
{ }^{\mathrm{CC}} \mathcal{D}_{a}^{v, \varrho} w(s) \in \breve{\mathfrak{R}}(s, w(s)) \quad(s \in[a, T], a \geq 0)
$$

subject to four-point conditions in the frame of the newly defined mixed RiemannLiouville conformable integro-derivative

$$
w(a)={ }^{\mathrm{RC}} \mathcal{D}_{a}^{v, p^{*}} w(\xi)+\mu_{1}, \quad w(T)={ }^{\mathrm{RC}} \mathcal{I}_{a}^{v, q^{*}} w(\sigma)+\mu_{2},
$$

so that ${ }^{\mathrm{CC}} \mathcal{D}_{a}^{v, \varrho}$ is the Caputo conformable derivative of fractional order $\varrho \in(1,2]$ with $v \in(0,1],{ }^{\mathrm{RC}} \mathcal{D}_{a}^{v, p^{*}}$ is the Riemann-Liouville conformable derivative of fractional order $p^{*} \in(0,1]$, and ${ }^{\mathrm{RC}} \mathcal{I}_{a}^{v, q^{*}}$ is the Riemann-Liouville conformable integral of fractional order $q^{*}>0$. Also $\xi, \sigma \in(a, T), \mu_{1}, \mu_{2} \in \mathbb{R}$ and $\breve{R}:[a, T] \times \mathbb{R} \rightarrow \mathcal{P}(\mathbb{R})$ is a set-valued map endowed with some properties which are stated in the sequel. Our main goal in the present manuscript is to obtain some existence criteria for the mentioned Caputo conformable differential inclusion. In this way, we employ some analytical techniques on the $\alpha-\psi$ contractive mappings and operators having the approximate endpoint property to reach the desired theoretical results. Note that unlike other published papers in the field of the existence theory, this inclusion problem supplemented with newly defined Caputo and Riemann-Liouville conformable operators is unique, and this type of mixed inclusion problems has not been investigated in any literature. We arrange the contents of the paper as follows. Some auxiliary definitions and notions are assembled in Sect. 2. Then in Sect. 3, we utilize two concepts of fixed point and endpoint to obtain the existence criteria 
corresponding to the given BVP by (1)-(2). In the last section, we propose an illustrative example to support our findings from a numerical point of view.

\section{Preliminaries}

In the current section, we state some fundamental and auxiliary concepts. As we know, the notion of the Riemann-Liouville integral of order $\varrho>0$ of a function $w:[0,+\infty) \rightarrow$ $\mathbb{R}$ is given by ${ }^{\mathrm{R}} \mathcal{I}_{0}^{\varrho} w(s)=\int_{0}^{s} \frac{(s-r)^{\varrho-1}}{\Gamma(\varrho)} w(r) \mathrm{d} r$ provided that the value of the integral is finite $[33,34]$. In this position, let us assume that $\varrho \in(n-1, n)$ so that $n=[\varrho]+1$. For a function $w \in \mathcal{A C}_{\mathbb{R}}^{(n)}([0,+\infty))$, the fractional derivative of Caputo type is given by

$$
{ }^{C} \mathcal{D}_{0}^{\varrho} w(s)=\int_{0}^{s} \frac{(s-r)^{n-\varrho-1}}{\Gamma(n-\varrho)} w^{(n)}(r) \mathrm{d} r
$$

provided that the integral is finite-valued [33, 34]. The left conformable derivative at the initial point $s_{0}=a$ for a function $w:[a, \infty) \rightarrow \mathbb{R}$ with $v \in(0,1]$ is defined as follows:

$$
\mathcal{D}_{a}^{v} w(s)=\lim _{\lambda \rightarrow 0} \frac{w\left(s+\lambda(s-a)^{1-v}\right)-w(s)}{\lambda},
$$

so that the limit exists [16]. It is notable that $\mathcal{D}_{a}^{v} w(a)=\lim _{s \rightarrow a^{+}} \mathcal{D}_{a}^{v} w(s)$ whenever $\mathcal{D}_{a}^{v} w(s)$ exists on the interval $(a, b)$. Moreover, it is obvious that $\mathcal{D}_{a}^{v} w(s)=(s-a)^{1-v} w^{\prime}(s)$ if the function $w$ is differentiable. Besides, the definition of the left conformable integral of $w$ with $v \in(0,1]$ is of the form $\mathcal{I}_{a}^{v} w(s)=\int_{a}^{s} w(r) \frac{\mathrm{d} r}{(r-a)^{1-v}}$ if the integral has finite values [16]. In [17], Jarad et al. extended the conformable operators to arbitrary orders in the Caputo and Riemann-Liouville setting. Assume that $\varrho \in \mathbb{C}$ with $\operatorname{Re}(\varrho) \geq 0$. Then the RiemannLiouville fractional conformable integral of a function $w$ of order $\varrho$ with $v \in(0,1]$ is defined by

$$
{ }^{\mathrm{RC}} \mathcal{I}_{a}^{v, \varrho} w(s)=\frac{1}{\Gamma(\varrho)} \int_{a}^{s}\left(\frac{(s-a)^{v}-(r-a)^{v}}{v}\right)^{\varrho-1} w(r) \frac{\mathrm{d} r}{(r-a)^{1-\varrho}}
$$

if the value of integral exists [17]. One can easily see that if $a=0$ and $v=1$, then ${ }^{\mathrm{RC}} \mathcal{I}_{a}^{v, \varrho} w(s)$ is reduced to the usual Riemann-Liouville integral ${ }^{\mathrm{R}} \mathcal{I}_{0}^{\varrho} w(s)$. On the other hand, the Riemann-Liouville conformable derivative of a function $w$ of order $\varrho$ with $v \in(0,1]$ is given by

$$
\begin{aligned}
{ }^{\mathrm{RC}} \mathcal{D}_{a}^{v, \varrho} w(s) & =\mathcal{D}_{a}^{v, n}\left({ }^{\mathrm{RC}} \mathcal{I}_{a}^{v, n-\varrho} w\right)(s) \\
& =\frac{\mathcal{D}_{a}^{v, n}}{\Gamma(n-\varrho)} \int_{a}^{s}\left(\frac{(s-a)^{v}-(r-a)^{v}}{v}\right)^{n-\varrho-1} w(r) \frac{\mathrm{d} r}{(r-a)^{1-\varrho}}
\end{aligned}
$$

so that $n=[\operatorname{Re}(\varrho)]+1$ and $\mathcal{D}_{a}^{v, n}=\overbrace{\mathcal{D}_{a}^{v} \mathcal{D}_{a}^{v} \cdots \mathcal{D}_{a}^{v}}^{n \text { times }}$, where $\mathcal{D}_{a}^{v}$ denotes the left conformable derivative with $v \in(0,1]$ [17]. Similarly, it is evident that if $a=0$ and $v=1$, then ${ }^{\mathrm{RC}} \mathcal{D}_{a}^{v, Q} w(s)$ is reduced to the usual Riemann-Liouville derivative ${ }^{\mathrm{R}} \mathcal{D}_{0}^{\varrho} w(s)$. Now, to define a similar notion in the Caputo setting, construct $\mathcal{L}_{v}(a):=\left\{\varphi:[a, b] \rightarrow \mathbb{R}: \mathcal{I}_{a}^{v} \varphi(s)\right.$ exists for any $s \in$ $[a, b]\}$ for $v \in(0,1]$ and set

$$
\mathbb{I}_{v}([a, b]):=\left\{w:[a, b] \rightarrow \mathbb{R}: w(s)=\mathcal{I}_{a}^{v} \varphi(s)+w(a) \text { for some } \varphi \in \mathcal{L}_{v}(a)\right\}
$$


where the notation $\mathcal{I}_{a}^{v} \varphi(s)=\int_{a}^{s} \varphi(r) \mathrm{d} v(r, a)=\int_{a}^{s} \varphi(r) \frac{\mathrm{d} r}{(r-a)^{1-v}}$ stands for the left conformable integral of $\varphi$ by putting $\mathrm{d} v(r, a)=\frac{\mathrm{d} r}{(r-a)^{1-v}}$ [15]. Also, if $a=0$, then we write $\mathrm{d} v(r)=$ $\frac{\mathrm{d} r}{r^{1-v}}$. For $n=1,2,3, \ldots$, we define $\mathcal{C}_{a, v}^{n}([a, b]):=\left\{w:[a, b] \rightarrow \mathbb{R}: \mathcal{D}_{a}^{v, n-1} w \in \mathbb{I}_{v}([a, b])\right\}$. Then the Caputo conformable derivative of a function $w \in \mathcal{C}_{a, v}^{n}([a, b])$ of order $\varrho$ with $v \in(0,1]$ is given by

$$
\begin{aligned}
{ }^{\mathrm{CC}} \mathcal{D}_{a}^{v, \varrho} w(s) & ={ }^{\mathrm{RC}} \mathcal{I}_{a}^{v, n-\varrho}\left(\mathcal{D}_{a}^{v, n} w\right)(s) \\
& =\frac{1}{\Gamma(n-\varrho)} \int_{a}^{s}\left(\frac{(s-a)^{v}-(r-a)^{v}}{v}\right)^{n-\varrho-1} \mathcal{D}_{a}^{v, n} w(r) \frac{\mathrm{d} r}{(r-a)^{1-\varrho}}
\end{aligned}
$$

so that $n=[\operatorname{Re}(\varrho)]+1$ [17]. Obviously, ${ }^{\mathrm{CC}} \mathcal{D}_{a}^{v, \varrho} w(s)={ }^{C} \mathcal{D}_{0}^{\varrho} w(s)$ if $a=0$ and $v=1$. Some important properties of the Caputo and Riemann-Liouville fractional conformable operators can be found in the following lemmas.

Lemma 1 ([17]) Assume that $\operatorname{Re}(\varrho)>0, \operatorname{Re}(\varpi)>0$, and $\operatorname{Re}(\beta)>0$. Then, for $v \in(0,1]$ and for all $s>a$, the following statements hold:

(i1) ${ }^{\mathrm{RC}} \mathcal{I}_{a}^{v, \varrho}\left({ }^{\mathrm{RC}} \mathcal{I}_{a}^{v, \varpi} w\right)(s)=\left({ }^{\mathrm{RC}} \mathcal{I}_{a}^{v, \varrho+\varpi} w\right)(s)$,

(i2) ${ }^{\mathrm{RC}} \mathcal{I}_{a}^{v, \varrho}(s-a)^{v(\beta-1)}(z)=\frac{1}{v^{\varrho}} \frac{\Gamma(\beta)}{\Gamma(\beta+\varrho)}(z-a)^{v(\beta+\varrho-1)}$,

(i3) ${ }^{\mathrm{RC}} \mathcal{D}_{a}^{v, \varrho}(s-a)^{v(\beta-1)}(z)=v^{\varrho} \frac{\Gamma(\beta)}{\Gamma(\beta-\varrho)}(z-a)^{v(\beta-\varrho-1)}$,

(i4) ${ }^{\mathrm{RC}} \mathcal{D}_{a}^{v, \varrho}\left({ }^{\mathrm{RC}} \mathcal{I}_{a}^{v, \varpi} w\right)(s)=\left({ }^{\mathrm{RC}} \mathcal{I}_{a}^{v, \varpi-\varrho} w\right)(s),(\operatorname{Re}(\varrho)<\operatorname{Re}(\varpi))$.

Lemma 2 ([19]) Let $n-1<\operatorname{Re}(\varrho) \leq n$ and $w \in \mathcal{C}_{a, v}^{n}([a, b])$. Then, for $v \in(0,1]$, we have

$$
{ }^{\mathrm{RC}} \mathcal{I}_{a}^{v, \varrho}\left({ }^{\mathrm{CC}} \mathcal{D}_{a}^{v, \varrho} w\right)(s)=w(s)-\sum_{j=0}^{n-1} \frac{\mathcal{D}_{a}^{v, j} w(a)}{v^{j} j !}(s-a)^{j v}
$$

In view of the above lemma, it is verified that the general solution of the homogeneous equation $\left({ }^{\mathrm{CC}} \mathcal{D}_{a}^{v, Q} w\right)(s)=0$ is given by

$$
w(s)=\sum_{j=0}^{n-1} b_{j}(s-a)^{j v}=b_{0}+b_{1}(s-a)^{v}+b_{2}(s-a)^{2 v}+\cdots+b_{n-1}(s-a)^{(n-1) v},
$$

where $n-1<\operatorname{Re}(\varrho) \leq n$ and $b_{0}, b_{1}, \ldots, b_{n-1} \in \mathbb{R}$.

In the following, we review some notions about the set-valued maps theory. For this purpose, consider the normed space $\left(\mathcal{W},\|\cdot\|_{\mathcal{W}}\right)$. Also, for convenience, we use the notations $\mathcal{P}(\mathcal{W}), \mathcal{P}_{\text {cls }}(\mathcal{W}), \mathcal{P}_{\text {bnd }}(\mathcal{W}), \mathcal{P}_{\text {cmp }}(\mathcal{W})$, and $\mathcal{P}_{\text {cvx }}(\mathcal{W})$ for the representation of the collection of all subsets, all closed subsets, all bounded subsets, all compact subsets, and all convex subsets of $\mathcal{W}$, respectively. The element $w^{*} \in \mathcal{W}$ is a fixed point for the given setvalued map $\breve{\mathfrak{R}}: \mathcal{W} \rightarrow \mathcal{P}(\mathcal{W})$ whenever $w^{*} \in \breve{\mathfrak{R}}\left(w^{*}\right)$ [35]. We represent the family of all fixed points of $\breve{\mathfrak{R}}$ by notation $\mathcal{F} \mathcal{I} \mathcal{X}(\breve{\mathfrak{R}})$ [35]. In the following, the Pompeiu-Hausdorff metric $\mathrm{PH}_{d_{\mathcal{W}}}: \mathcal{P}(\mathcal{W}) \times \mathcal{P}(\mathcal{W}) \rightarrow \mathbb{R}^{*}=\mathbb{R} \cup\{\infty\}$ is formulated by

$$
\mathrm{PH}_{d_{\mathcal{W}}}\left(A_{1}, A_{2}\right)=\max \left\{\sup _{a_{1} \in A_{1}} d_{\mathcal{W}}\left(a_{1}, A_{2}\right), \sup _{a_{2} \in A_{2}} d_{\mathcal{W}}\left(A_{1}, a_{2}\right)\right\},
$$

where $d_{\mathcal{W}}\left(A_{1}, a_{2}\right)=\inf _{a_{1} \in A_{1}} d_{\mathcal{W}}\left(a_{1}, a_{2}\right)$ and $d_{\mathcal{W}}\left(a_{1}, A_{2}\right)=\inf _{a_{2} \in A_{2}} d_{\mathcal{W}}\left(a_{1}, a_{2}\right)$ [35]. A setvalued map $\breve{\mathfrak{R}}: \mathcal{W} \rightarrow \mathcal{P}_{\text {cls }}(\mathcal{W})$ is Lipschitzian with positive constant $\hat{\lambda}$ if the inequality 
$\mathrm{PH}_{d_{\mathcal{W}}}\left(\breve{\mathfrak{R}}(w), \breve{\mathfrak{R}}\left(w^{\prime}\right)\right) \leq \hat{\lambda} d_{\mathcal{W}}\left(w, w^{\prime}\right)$ holds for all $w, w^{\prime} \in \mathcal{W}$. A Lipschitz map $\breve{\mathfrak{R}}$ is supposed to be defined contraction if $\hat{\lambda} \in(0,1)$ [35]. In the sequel, $\breve{\mathfrak{R}}$ is said to be completely continuous if $\breve{\mathfrak{R}}(K)$ is relatively compact for each $K \in \mathcal{P}_{\text {bnd }}(\mathcal{W})$, whereas $\breve{\mathfrak{R}}:[0,1] \rightarrow \mathcal{P}_{\text {cls }}(\mathbb{R})$ is called measurable if $s \longmapsto d_{\mathcal{W}}(v, \breve{\mathfrak{R}}(s))$ is measurable for any $v \in \mathbb{R}[35,36]$. Also, $\breve{\mathfrak{R}}$ is upper semi-continuous whenever for every $w^{*} \in \mathcal{W}$ the set $\breve{\mathfrak{R}}\left(w^{*}\right)$ belongs to $\mathcal{P}_{\text {cls }}(\mathcal{W})$ and for each open set $\mathcal{U}$ containing $\breve{\mathfrak{R}}\left(w^{*}\right)$ there is a neighborhood $\mathcal{O}_{0}^{*}$ of $w^{*}$ provided that $\breve{\mathfrak{R}}\left(\mathcal{O}_{0}^{*}\right) \subseteq \mathcal{U}$ [35]. We construct a graph of the set-valued map $\breve{\mathfrak{R}}: \mathcal{W} \rightarrow \mathcal{P}_{\text {cls }}(\mathcal{Z})$ by $\operatorname{Graph}(\breve{\mathfrak{R}})=\{(w, z) \in \mathcal{W} \times \mathcal{Z}: z \in \breve{\mathfrak{R}}(w)\}$. The $\operatorname{Graph}(\breve{\mathfrak{R}})$ is closed whenever, for two arbitrary convergent sequences $\left\{w_{n}\right\}_{n \geq 1}$ in $\mathcal{W}$ and $\left\{z_{n}\right\}_{n \geq 1}$ in $\mathcal{Z}$ with $w_{n} \rightarrow w_{0}, z_{n} \rightarrow z_{0}$ and $z_{n} \in \breve{\mathfrak{R}}\left(w_{n}\right)$, we have the inclusion $z_{0} \in \breve{\mathfrak{R}}\left(w_{0}\right)$ [35, 36]. In view of [35], it is deduced that if the set-valued map $\breve{\mathfrak{R}}: \mathcal{W} \rightarrow \mathcal{P}_{\text {cls }}(\mathcal{Z})$ has an upper semi-continuity property, then $\operatorname{Graph}(\breve{\mathfrak{R}})$ is a closed subset of $\mathcal{W} \times \mathcal{Z}$. On the contrary, if $\breve{\mathfrak{R}}$ has the complete continuity and closed graph property, then $\breve{\mathfrak{R}}$ is upper semi-continuous [35]. In addition, $\breve{\mathfrak{R}}$ has convex values if $\breve{\mathfrak{R}}(k) \in \mathcal{P}_{\text {cvx }}(\mathcal{W})$ for each $w \in \mathcal{W}$. Furthermore, a collection of selections of $\breve{\Re}$ at point $w \in \mathcal{C}_{\mathbb{R}}([0,1])$ is represented by

$$
(\mathfrak{S E L})_{\breve{\Re}, w}:=\left\{\hat{\vartheta} \in \mathcal{L}_{\mathbb{R}}^{1}([0,1]): \hat{\vartheta}(s) \in \breve{\mathfrak{R}}(s, w(s))\right\}
$$

for (a.e.) all $s \in[0,1][35,36]$. Note that if we assume that $\breve{\mathfrak{R}}$ is an arbitrary set-valued map, then for each $w \in \mathcal{C}_{\mathcal{W}}([0,1])$ we have $(\mathfrak{S E L})_{\breve{R}, w} \neq \emptyset$ whenever $\operatorname{dim}(\mathcal{W})<\infty$ [35]. We say that $\breve{\mathfrak{R}}:[0,1] \times \mathbb{R} \rightarrow \mathcal{P}(\mathbb{R})$ is called Caratheodory if $s \mapsto \breve{\mathfrak{R}}(s, w)$ is measurable for every $w \in \mathbb{R}$ and $w \mapsto \breve{\mathfrak{R}}(s, w)$ is upper semi-continuous for (a.e.) all $w \in[0,1][35,36]$. Besides, a Caratheodory set-valued map $\breve{\mathfrak{R}}:[0,1] \times \mathbb{R} \rightarrow \mathcal{P}(\mathbb{R})$ is called $\mathcal{L}^{1}$-Caratheodory when, for each $\mu>0$, there is $\varphi_{\mu} \in \mathcal{L}_{\mathbb{R}^{+}}^{1}([0,1])$ so that $\|\breve{\mathfrak{R}}(s, w)\|=\sup _{s \in[0,1]}\{|q|: q \in \breve{\mathfrak{R}}(s, w)\} \leq \varphi_{\mu}(s)$ for all $|w| \leq \mu$ and for almost any $s \in[0,1][35,36]$.

Samet et al. [37] introduced a new collection of nondecreasing and nonnegative functions $\psi:[0, \infty) \rightarrow[0, \infty)$ with $\sum_{n=1}^{\infty} \psi^{n}(s)<\infty$ which is represented by $\Psi$. By considering the properties of these functions, it is obvious that $\psi(s)<s$ for all $s>0$ [37]. Later, Mohammadi et al. constructed a new structure for set-valued maps with the following definition [38]. A set-valued map $\breve{\mathfrak{R}}: \mathcal{W} \rightarrow \mathcal{P}_{\text {cls,bnd }}(\mathcal{W})$ is said to be $\alpha-\psi$-contraction if

$$
\alpha\left(w, w^{\prime}\right) \mathrm{PH}_{d_{\mathcal{W}}}\left(\breve{\mathfrak{R}} w, \breve{\mathfrak{R}} w^{\prime}\right) \leq \psi\left(d_{\mathcal{W}}\left(w, w^{\prime}\right)\right)
$$

for each $w, w^{\prime} \in \mathcal{W}$ [38]. In addition, we say that $\mathcal{W}$ has the property $\left(C_{\alpha}\right)$ if for every convergent sequence $\left\{w_{n}\right\}$ in $\mathcal{W}$ with $w_{n} \rightarrow w$ and $\alpha\left(w_{n}, w_{n+1}\right) \geq 1$ for any $n \in \mathbb{N}$ there is a subsequence $\left\{w_{n_{j}}\right\}$ of $\left\{w_{n}\right\}$ such that $\alpha\left(w_{n_{j}}, w\right) \geq 1$ for each $j \in \mathbb{N}$. Also $\breve{\mathfrak{R}}$ is called $\alpha$-admissible if for each $w \in \mathcal{W}$ and $w^{\prime} \in \breve{\mathfrak{R}}(w)$ with $\alpha\left(w, w^{\prime}\right) \geq 1$ we have $\alpha\left(w^{\prime}, w^{\prime \prime}\right) \geq 1$ for all $w^{\prime \prime} \in \breve{\mathfrak{R}}\left(w^{\prime}\right)$ [38]. Finally, $w \in \mathcal{W}$ is called the endpoint of $\breve{\mathfrak{R}}: \mathcal{W} \rightarrow \mathcal{P}(\mathcal{W})$ if $\breve{\mathfrak{R}}(w)=\{w\}$ [39]. Besides, we say that $\mathcal{S}$ has an approximate endpoint property if we have $\inf _{w \in \mathcal{W}} \sup _{z \in \breve{\Re} w} d_{\mathcal{W}}(w, z)=0$ [39]. The following theorems are our required tool for establishing the desired results in this research.

Theorem 3 ([40]) Let $\mathcal{W}$ be a separable Banach space, $\breve{\mathfrak{R}}:[0,1] \times \mathcal{W} \rightarrow \mathcal{P}_{\text {cmp,cvx }}(\mathcal{W})$ be an $\mathcal{L}^{1}$-Carathéodory set-valued map, and $\Xi: \mathcal{L}_{\mathcal{W}}^{1}([0,1]) \rightarrow \mathcal{C}_{\mathcal{W}}([0,1])$ be a linear continuous map. Then the composition $\Xi \circ(\mathfrak{S E E})_{\mathfrak{R}}: \mathcal{C}_{\mathcal{W}}([0,1]) \rightarrow \mathcal{P}_{\text {cmp,cvx }}\left(\mathcal{C}_{\mathcal{W}}([0,1])\right)$ is a new oper-

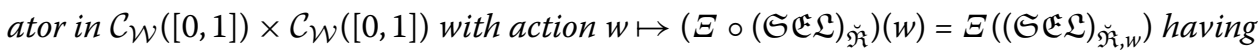
the closed graph property. 
Theorem 4 ([38]) Let $\left(\mathcal{W}, d_{\mathcal{W}}\right)$ be a complete metric space. Assume that $\alpha$ is a nonnegative map on $\mathcal{W} \times \mathcal{W}, \psi \in \Psi$ is a strictly increasing map, and $\breve{\mathfrak{R}}: \mathcal{W} \rightarrow \mathcal{P}_{\text {cls,bnd }}(\mathcal{W})$ is an $\alpha$ admissible and $\alpha$ - $\psi$-contractive set-valued map so that $\alpha\left(w, w^{\prime}\right) \geq 1$ for some $w \in \mathcal{W}$ and $w^{\prime} \in \breve{\mathfrak{R}}(w)$. Then $\breve{\mathfrak{R}}$ has a fixed point whenever the space $\mathcal{W}$ has the property $\left(C_{\alpha}\right)$.

Theorem 5 ([39]) Let $\left(\mathcal{W}, d_{\mathcal{W}}\right)$ be a complete metric space and $\psi:[0, \infty) \rightarrow[0, \infty)$ be upper semi-continuous provided that $\psi(s)<s$ and $\liminf _{s \rightarrow \infty}(s-\psi(s))>0$ for each $s>0$. Also, suppose that $\breve{\mathfrak{R}}: \mathcal{W} \rightarrow \mathcal{P}_{\text {cls,bnd }}(\mathcal{W})$ is such that $\mathrm{PH}_{d_{\mathcal{W}}}\left(\breve{\mathfrak{R}} w, \breve{\mathfrak{R}} w^{\prime}\right) \leq \psi\left(d_{\mathcal{W}}\left(w, w^{\prime}\right)\right)$ for each $w, w^{\prime} \in \mathcal{W}$. Then $\breve{\mathfrak{R}}$ has a unique endpoint iff $\breve{\mathfrak{R}}$ has the approximate endpoint property.

\section{Main results}

Consider the sup norm $\|w\|_{\mathcal{W}}=\sup _{s \in[a, T]}|w(s)|$ on the space $\mathcal{W}=\left\{w(s): w(s) \in \mathcal{C}_{\mathbb{R}}([a, T])\right\}$. Then $\left(\mathcal{W},\|\cdot\|_{\mathcal{W}}\right)$ is a Banach space. Further, consider the following constants for convenience:

$$
\begin{array}{ll}
k_{0}:=1-\frac{v^{p^{*}}(\xi-a)^{-v p^{*}}}{\Gamma\left(1-p^{*}\right)}, & k_{1}:=\frac{v^{p^{*}}(\xi-a)^{v\left(1-p^{*}\right)}}{\Gamma\left(2-p^{*}\right)}, \\
k_{2}:=\frac{(\sigma-a)^{v q^{*}}}{v^{q^{*}} \Gamma\left(1+q^{*}\right)}-1, & k_{3}:=\frac{(\sigma-a)^{v\left(1+q^{*}\right)}}{v^{q^{*}} \Gamma\left(2+q^{*}\right)}-(T-a)^{v} .
\end{array}
$$

In the following lemma, we introduce an equivalent integral structure for the solution of the four-point Caputo conformable inclusion BVP (1)-(2).

Lemma 6 Let $\hat{h} \in \mathcal{W}$. Then $w_{0}$ is a solution function for the Caputo conformable differential equation

$$
{ }^{\mathrm{CC}} \mathcal{D}_{a}^{v, \varrho} w(s)=\hat{h}(s) \quad(s \in[a, T], a \geq 0)
$$

with four-point mixed Riemann-Liouville conformable integro-derivative boundary conditions

$$
w(a)={ }^{\mathrm{RC}} \mathcal{D}_{a}^{v, p^{*}} w(\xi)+\mu_{1}, \quad w(T)={ }^{\mathrm{RC}} \mathcal{I}_{a}^{v, q^{*}} w(\sigma)+\mu_{2},
$$

if and only if $w_{0}$ satisfies the integral equation

$$
\begin{aligned}
w(s)= & \frac{1}{\Gamma(\varrho)} \int_{a}^{s}\left(\frac{(s-a)^{v}-(r-a)^{v}}{v}\right)^{\varrho-1} \hat{h}(r) \frac{\mathrm{d} r}{(r-a)^{1-v}} \\
& +\tilde{\Omega}_{1}(s) \frac{1}{\Gamma(\varrho)} \int_{a}^{T}\left(\frac{(T-a)^{v}-(r-a)^{v}}{v}\right)^{\varrho-1} \hat{h}(r) \frac{\mathrm{d} r}{(r-a)^{1-v}} \\
& +\tilde{\Omega}_{2}(s) \frac{1}{\Gamma\left(\varrho-p^{*}\right)} \int_{a}^{\xi}\left(\frac{(\xi-a)^{v}-(r-a)^{v}}{v}\right)^{\varrho-p^{*}-1} \hat{h}(r) \frac{\mathrm{d} r}{(r-a)^{1-v}} \\
& +\tilde{\Omega}_{3}(s) \frac{1}{\Gamma\left(\varrho+q^{*}\right)} \int_{a}^{\sigma}\left(\frac{(\sigma-a)^{v}-(r-a)^{v}}{v}\right)^{\varrho+q^{*}-1} \hat{h}(r) \frac{\mathrm{d} r}{(r-a)^{1-v}}+\tilde{\Omega}_{4}(s),
\end{aligned}
$$


where $\tilde{\Omega}_{1}(s), \tilde{\Omega}_{2}(s), \tilde{\Omega}_{3}(s)$, and $\tilde{\Omega}_{4}(s)$ are four functions with respect to $s$ which are defined in the following form:

$$
\begin{aligned}
& \tilde{\Omega}_{1}(s):=\frac{1}{k}\left(k_{1}+k_{0}(s-a)^{v}\right), \quad \tilde{\Omega}_{2}(s):=\frac{1}{k}\left(k_{3}-k_{2}(s-a)^{v}\right), \\
& \tilde{\Omega}_{3}(s):=\frac{1}{k}\left(k_{1}+k_{0}(s-a)^{v}\right), \\
& \tilde{\Omega}_{4}(s):=\frac{1}{k}\left(\mu_{1}\left(k_{3}-k_{2}(s-a)^{v}\right)-\mu_{2}\left(k_{1}+k_{0}(s-a)^{v}\right)\right),
\end{aligned}
$$

so that $k=k_{0} k_{3}+k_{1} k_{2} \neq 0$.

Proof In the beginning, $w_{0}$ is supposed to satisfy the Caputo conformable equation (4). Clearly, we have ${ }^{{ }^{C C}} \mathcal{D}_{a}^{v, Q} w_{0}(s)=\hat{h}(s)$. Now, by taking $\varrho$ th order Riemann-Liouville conformable integral on the last equality, we obtain the following equation:

$$
w_{0}(s)=\frac{1}{\Gamma(\varrho)} \int_{a}^{s}\left(\frac{(s-a)^{v}-(r-a)^{v}}{v}\right)^{\varrho-1} \hat{h}(r) \frac{\mathrm{d} r}{(r-a)^{1-v}}+b_{0}+b_{1}(s-a)^{v}
$$

where we wish to seek two constants $b_{0}, b_{1} \in \mathbb{R}$. On the other hand, by taking the Riemann-Liouville conformable derivative and integral with respect to $s$ on both sides of equation (8), we get

$$
\begin{aligned}
{ }^{\mathrm{RC}} \mathcal{D}_{a}^{v, p^{*}} w_{0}(s)= & \frac{1}{\Gamma\left(\varrho-p^{*}\right)} \int_{a}^{s}\left(\frac{(s-a)^{v}-(r-a)^{v}}{v}\right)^{\varrho-p^{*}-1} \hat{h}(r) \frac{\mathrm{d} r}{(r-a)^{1-v}} \\
& +b_{0} \frac{v^{p^{*}}(s-a)^{-v p^{*}}}{\Gamma\left(1-p^{*}\right)}+b_{1} \frac{v^{p^{*}}(s-a)^{v\left(1-p^{*}\right)}}{\Gamma\left(2-p^{*}\right)}
\end{aligned}
$$

and

$$
\begin{aligned}
{ }^{\mathrm{RC}} \mathcal{I}_{a}^{v, q^{*}} w_{0}(s)= & \frac{1}{\Gamma\left(\varrho+q^{*}\right)} \int_{a}^{s}\left(\frac{(s-a)^{v}-(r-a)^{v}}{v}\right)^{\varrho+q^{*}-1} \hat{h}(r) \frac{\mathrm{d} r}{(r-a)^{1-v}} \\
& +b_{0} \frac{(s-a)^{v q^{*}}}{v^{q^{*}} \Gamma\left(1+q^{*}\right)}+b_{1} \frac{(s-a)^{v\left(1+q^{*}\right)}}{v^{q^{*}} \Gamma\left(2+q^{*}\right)} .
\end{aligned}
$$

By applying the four-point mixed boundary conditions, we get

$$
\begin{aligned}
k_{0} b_{0} & -k_{1} b_{1} \\
= & \frac{1}{\Gamma\left(\varrho-p^{*}\right)} \int_{a}^{\xi}\left(\frac{(\xi-a)^{v}-(r-a)^{v}}{v}\right)^{\varrho-p^{*}-1} \hat{h}(r) \frac{\mathrm{d} r}{(r-a)^{1-v}}+\mu_{1}
\end{aligned}
$$

and

$$
\begin{aligned}
k_{2} b_{0}+k_{3} b_{1}= & \frac{1}{\Gamma(\varrho)} \int_{a}^{T}\left(\frac{(T-a)^{v}-(r-a)^{v}}{v}\right)^{\varrho-1} \hat{h}(r) \frac{\mathrm{d} r}{(r-a)^{1-v}} \\
& -\frac{1}{\Gamma\left(\varrho+q^{*}\right)} \int_{a}^{\sigma}\left(\frac{(\sigma-a)^{v}-(r-a)^{v}}{v}\right)^{\varrho+q^{*}-1} \hat{h}(r) \frac{\mathrm{d} r}{(r-a)^{1-v}}+\mu_{2},
\end{aligned}
$$


where $k_{0}, k_{1}, k_{2}, k_{3}$ are given in (3). By some direct computations on equations (9) and (10), we obtain

$$
\begin{aligned}
b_{0}= & \frac{1}{k} \\
& \times\left[k_{3}\left(\frac{1}{\Gamma\left(\varrho-p^{*}\right)} \int_{a}^{\xi}\left(\frac{(\xi-a)^{v}-(r-a)^{v}}{v}\right)^{\varrho-p^{*}-1} \hat{h}(r) \frac{\mathrm{d} r}{(r-a)^{1-v}}+\mu_{1}\right)\right. \\
& +k_{1}\left(\frac{1}{\Gamma(\varrho)} \int_{a}^{T}\left(\frac{(T-a)^{v}-(r-a)^{v}}{v}\right)^{\varrho-1} \hat{h}(r) \frac{\mathrm{d} r}{(r-a)^{1-v}}\right. \\
& \left.\left.-\frac{1}{\Gamma\left(\varrho+q^{*}\right)} \int_{a}^{\sigma}\left(\frac{(\sigma-a)^{v}-(r-a)^{v}}{v}\right)^{\varrho+q^{*}-1} \hat{h}(r) \frac{\mathrm{d} r}{(r-a)^{1-v}}-\mu_{2}\right)\right]
\end{aligned}
$$

and

$$
\begin{aligned}
b_{1}= & \frac{1}{k} \\
& \times\left[-k_{2}\left(\frac{1}{\Gamma\left(\varrho-p^{*}\right)} \int_{a}^{\xi}\left(\frac{(\xi-a)^{v}-(r-a)^{v}}{v}\right)^{\varrho-p^{*}-1} \hat{h}(r) \frac{\mathrm{d} r}{(r-a)^{1-v}}+\mu_{1}\right)\right. \\
& +k_{0}\left(\frac{1}{\Gamma(\varrho)} \int_{a}^{T}\left(\frac{(T-a)^{v}-(r-a)^{v}}{v}\right)^{\varrho-1} \hat{h}(r) \frac{\mathrm{d} r}{(r-a)^{1-v}}\right. \\
& \left.\left.-\frac{1}{\Gamma\left(\varrho+q^{*}\right)} \int_{a}^{\sigma}\left(\frac{(\sigma-a)^{v}-(r-a)^{v}}{v}\right)^{\varrho+q^{*}-1} \hat{h}(r) \frac{\mathrm{d} r}{(r-a)^{1-v}}-\mu_{2}\right)\right] .
\end{aligned}
$$

If we insert the values $b_{0}$ and $b_{1}$ into equation (8), then we have

$$
\begin{aligned}
w_{0}(s)= & \frac{1}{\Gamma(\varrho)} \int_{a}^{s}\left(\frac{(s-a)^{v}-(r-a)^{v}}{v}\right)^{\varrho-1} \hat{h}(r) \frac{\mathrm{d} r}{(r-a)^{1-v}} \\
& +\tilde{\Omega}_{1}(s) \frac{1}{\Gamma(\varrho)} \int_{a}^{T}\left(\frac{(T-a)^{v}-(r-a)^{v}}{v}\right)^{\varrho-1} \hat{h}(r) \frac{\mathrm{d} r}{(r-a)^{1-v}} \\
& +\tilde{\Omega}_{2}(s) \frac{1}{\Gamma\left(\varrho-p^{*}\right)} \int_{a}^{\xi}\left(\frac{(\xi-a)^{v}-(r-a)^{v}}{v}\right)^{\varrho-p^{*}-1} \hat{h}(r) \frac{\mathrm{d} r}{(r-a)^{1-v}} \\
& +\tilde{\Omega}_{3}(s) \frac{1}{\Gamma\left(\varrho+q^{*}\right)} \int_{a}^{\sigma}\left(\frac{(\sigma-a)^{v}-(r-a)^{v}}{v}\right)^{\varrho+q^{*}-1} \hat{h}(r) \frac{\mathrm{d} r}{(r-a)^{1-v}}+\tilde{\Omega}_{4}(s)
\end{aligned}
$$

which shows that the function $w_{0}$ satisfies the integral equation (6). In the opposite direction, one can easily check that $w_{0}$ is a solution for the four-point Caputo conformable BVP (4)-(5) whenever $w_{0}$ satisfies the integral equation (6).

For the sake of convenience in writing, we set

$$
\begin{aligned}
& \left|\tilde{\Omega}_{1}(s)\right| \leq \Omega_{1}^{*}:=\frac{1}{|k|}\left(\left|k_{1}\right|+\left|k_{0}\right|(T-a)^{v}\right), \\
& \left|\tilde{\Omega}_{2}(s)\right| \leq \Omega_{2}^{*}:=\frac{1}{|k|}\left(\left|k_{3}\right|+\left|k_{2}\right|(T-a)^{v}\right), \\
& \left|\tilde{\Omega}_{3}(s)\right| \leq \Omega_{3}^{*}:=\frac{1}{|k|}\left(\left|k_{1}\right|+\left|k_{0}\right|(T-a)^{v}\right),
\end{aligned}
$$




$$
\begin{aligned}
& \left|\tilde{\Omega}_{4}(s)\right| \leq \Omega_{4}^{*}:=\frac{1}{|k|}\left(\left|\mu_{1}\right|\left(\left|k_{3}\right|+\left|k_{2}\right|(T-a)^{v}\right)+\left|\mu_{2}\right|\left(\left|k_{1}\right|+\left|k_{0}\right|(T-a)^{v}\right)\right) \\
& M=\frac{(T-a)^{v \varrho}}{v^{\varrho} \Gamma(\varrho+1)}+\Omega_{1}^{*} \frac{(T-a)^{v}}{v^{\varrho} \Gamma(\varrho+1)} \\
& \quad+\Omega_{2}^{*} \frac{(\xi-a)^{v\left(\varrho-p^{*}\right)}}{v^{\varrho-p^{*}} \Gamma\left(\varrho-p^{*}+1\right)}+\Omega_{3}^{*} \frac{(\sigma-a)^{v\left(\varrho+q^{*}\right)}}{v^{\varrho+q^{*}} \Gamma\left(\varrho+q^{*}+1\right)}
\end{aligned}
$$

Definition 7 The function $w \in \mathcal{A C}_{\mathbb{R}}([a, T])$ is considered as a solution for the four-point Caputo conformable inclusion BVP (1)-(2) if there is an integrable function $\hat{\vartheta} \in \mathcal{L}_{\mathbb{R}}^{1}([a, T])$ with $\hat{\vartheta} \in \breve{\mathfrak{R}}(s, w(s))$ for almost all $s \in[a, T]$ satisfying the four-point mixed RiemannLiouville conformable integro-derivative conditions

$$
w(a)={ }^{\mathrm{RC}} \mathcal{D}_{a}^{v, p^{*}} w(\xi)+\mu_{1}, \quad w(T)={ }^{\mathrm{RC}} \mathcal{I}_{a}^{v, q^{*}} w(\sigma)+\mu_{2}
$$

and

$$
\begin{aligned}
w(s)= & \frac{1}{\Gamma(\varrho)} \int_{a}^{s}\left(\frac{(s-a)^{v}-(r-a)^{v}}{v}\right)^{\varrho-1} \hat{\vartheta}(r) \frac{\mathrm{d} r}{(r-a)^{1-v}} \\
& +\tilde{\Omega}_{1}(s) \frac{1}{\Gamma(\varrho)} \int_{a}^{T}\left(\frac{(T-a)^{v}-(r-a)^{v}}{v}\right)^{\varrho-1} \hat{\vartheta}(r) \frac{\mathrm{d} r}{(r-a)^{1-v}} \\
& +\tilde{\Omega}_{2}(s) \frac{1}{\Gamma\left(\varrho-p^{*}\right)} \int_{a}^{\xi}\left(\frac{(\xi-a)^{v}-(r-a)^{v}}{v}\right)^{\varrho-p^{*}-1} \hat{\vartheta}(r) \frac{\mathrm{d} r}{(r-a)^{1-v}} \\
& +\tilde{\Omega}_{3}(s) \frac{1}{\Gamma\left(\varrho+q^{*}\right)} \int_{a}^{\sigma}\left(\frac{(\sigma-a)^{v}-(r-a)^{v}}{v}\right)^{\varrho+q^{*}-1} \hat{\vartheta}(r) \frac{\mathrm{d} r}{(r-a)^{1-v}}+\tilde{\Omega}_{4}(s)
\end{aligned}
$$

for any $s \in[a, T]$.

In this position, for each $w \in \mathcal{W}$, we represent the family of selections of $\breve{\mathfrak{R}}$ as follows:

$$
(\mathfrak{S E L})_{\mathfrak{\Re}, w}=\left\{\hat{\vartheta} \in L^{1}([a, T]): \hat{\vartheta}(s) \in \breve{\mathfrak{R}}(s, w(s))\right\}
$$

for almost all $s \in[a, T]$. Next, we formulate the set-valued operator $\mathcal{K}: \mathcal{W} \rightarrow \mathcal{P}(\mathcal{W})$ by

$$
\mathcal{K}(w)=\left\{z \in \mathcal{W}: \text { there is } \hat{\vartheta} \in(\mathfrak{S E L})_{\breve{\mathfrak{R}}, w} \text { so that } z(s)=h(s) \text { for any } s \in[a, T]\right\},
$$

provided that

$$
\begin{aligned}
h(s)= & \frac{1}{\Gamma(\varrho)} \int_{a}^{s}\left(\frac{(s-a)^{v}-(r-a)^{v}}{v}\right)^{\varrho-1} \hat{\vartheta}(r) \frac{\mathrm{d} r}{(r-a)^{1-v}} \\
& +\tilde{\Omega}_{1}(s) \frac{1}{\Gamma(\varrho)} \int_{a}^{T}\left(\frac{(T-a)^{v}-(r-a)^{v}}{v}\right)^{\varrho-1} \hat{\vartheta}(r) \frac{\mathrm{d} r}{(r-a)^{1-v}} \\
& +\tilde{\Omega}_{2}(s) \frac{1}{\Gamma\left(\varrho-p^{*}\right)} \int_{a}^{\xi}\left(\frac{(\xi-a)^{v}-(r-a)^{v}}{v}\right)^{\varrho-p^{*}-1} \hat{\vartheta}(r) \frac{\mathrm{d} r}{(r-a)^{1-v}} \\
& +\tilde{\Omega}_{3}(s) \frac{1}{\Gamma\left(\varrho+q^{*}\right)} \int_{a}^{\sigma}\left(\frac{(\sigma-a)^{v}-(r-a)^{v}}{v}\right)^{\varrho+q^{*}-1} \hat{\vartheta}(r) \frac{\mathrm{d} r}{(r-a)^{1-v}}+\tilde{\Omega}_{4}(s) .
\end{aligned}
$$


Theorem 8 Let us suppose that $\breve{\mathfrak{R}}:[a, T] \times \mathcal{W} \rightarrow \mathcal{P}_{\text {cmp }}(\mathcal{W})$ is a compact set-valued map Furthermore, assume that all six assumptions are valid:

(Hp1) $\breve{\mathfrak{R}}$ is a bounded and integrable operator, and also the set $\breve{\mathfrak{R}}(\cdot, w):[a, T] \rightarrow \mathcal{P}_{\text {cmp }}(\mathcal{W})$ is measurable for each $w \in \mathcal{W} ;$

(Hp2) There are $\psi \in \Psi$ and $\breve{a} \in \mathcal{C}_{\mathbb{R} \geq 0}([a, T])$ such that

$$
\operatorname{PH}_{d_{\mathcal{W}}}\left(\breve{\mathfrak{R}}(s, w), \breve{\mathfrak{R}}\left(s, w^{\prime}\right)\right) \leq \breve{a}(s) \psi\left(\left|w-w^{\prime}\right|\right) \frac{1}{M\|\breve{a}\|}
$$

for all $s \in[a, T]$ and $w, w^{\prime} \in \mathcal{W}$, where $\sup _{s \in[a, T]}|\breve{a}(s)|=\|\breve{a}\|$ and the constant $M$ is given by (11);

(Hp3) There is a function $\tilde{\zeta}: \mathbb{R} \times \mathbb{R} \rightarrow \mathbb{R}$ provided that $\tilde{\zeta}\left(w, w^{\prime}\right) \geq 0$ for each $w, w^{\prime} \in \mathcal{W}$;

(Hp4) If the sequence $\left\{w_{n}\right\}_{n \geq 1} \subseteq \mathcal{W}$ converges to $w$ and $\tilde{\zeta}\left(w_{n}(s), w_{n+1}(s)\right) \geq 0$ for all $s \in[a, T]$ and $n \geq 1$, then there is a subsequence $\left\{w_{n_{l}}\right\}_{l \geq 1}$ of $\left\{w_{n}\right\}$ such that $\tilde{\zeta}\left(w_{n l}(s), w(s)\right) \geq 0$ for each $s \in[a, T]$ and $l \geq 1$;

(Hp5) There are two elements $w_{0} \in \mathcal{W}$ and $z \in \mathcal{K}\left(w_{0}\right)$ such that $\tilde{\zeta}\left(w_{0}(s), z(s)\right) \geq 0$ for all $s \in[a, T]$, where $\mathcal{K}: \mathcal{W} \rightarrow \mathcal{P}(\mathcal{W})$ is the same operator defined by (12);

(Hp6) For each $w \in \mathcal{W}$ and $z \in \mathcal{K}(w)$ with $\tilde{\zeta}(w(s), z(s)) \geq 0$, there is $h \in \mathcal{K}(w)$ so that $\tilde{\zeta}(z(s), h(s)) \geq 0$ for all $s \in[a, T]$.

Then the four-point Caputo conformable inclusion BVP (1)-(2) has a solution on $[a, T]$.

Proof It is a well-known fact that the solution for the four-point Caputo conformable inclusion BVP (1)-(2) is as a fixed point of the operator $\mathcal{K}: \mathcal{W} \rightarrow \mathcal{P}(\mathcal{W})$ given by (12). From assumption (Hp1), the measurability of the set-valued map $s \mapsto \breve{\mathfrak{R}}(s, w(s))$ is evident, and thus it is closed-valued for each $w \in \mathcal{W}$. Hence, $\breve{\mathfrak{R}}$ has measurable selection and $(\mathfrak{S E L})_{\breve{R}, w} \neq \emptyset$. Here, it is suitable to prove that $\mathcal{K}(w)$ is a closed subset of $\mathcal{W}$ for each $w \in \mathcal{W}$. To do this, we consider a sequence $\left\{w_{n}\right\}_{n \geq 1}$ of $\mathcal{K}(w)$ having the property $w_{n} \rightarrow w$. For each $n$, choose $\hat{\vartheta}_{n} \in(\mathfrak{S E L})_{\breve{\mathfrak{R}}, w}$ provided that

$$
\begin{aligned}
w_{n}(s)= & \frac{1}{\Gamma(\varrho)} \int_{a}^{s}\left(\frac{(s-a)^{v}-(r-a)^{v}}{v}\right)^{\varrho-1} \hat{\vartheta}_{n}(r) \frac{\mathrm{d} r}{(r-a)^{1-v}} \\
& +\tilde{\Omega}_{1}(s) \frac{1}{\Gamma(\varrho)} \int_{a}^{T}\left(\frac{(T-a)^{v}-(r-a)^{v}}{v}\right)^{\varrho-1} \hat{\vartheta}_{n}(r) \frac{\mathrm{d} r}{(r-a)^{1-v}} \\
& +\tilde{\Omega}_{2}(s) \frac{1}{\Gamma\left(\varrho-p^{*}\right)} \int_{a}^{\xi}\left(\frac{(\xi-a)^{v}-(r-a)^{v}}{v}\right)^{\varrho-p^{*}-1} \hat{\vartheta}_{n}(r) \frac{\mathrm{d} r}{(r-a)^{1-v}} \\
& +\tilde{\Omega}_{3}(s) \frac{1}{\Gamma\left(\varrho+q^{*}\right)} \\
& \times \int_{a}^{\sigma}\left(\frac{(\sigma-a)^{v}-(r-a)^{v}}{v}\right)^{\varrho+q^{*}-1} \hat{\vartheta}_{n}(r) \frac{\mathrm{d} r}{(r-a)^{1-v}}+\tilde{\Omega}_{4}(s)
\end{aligned}
$$

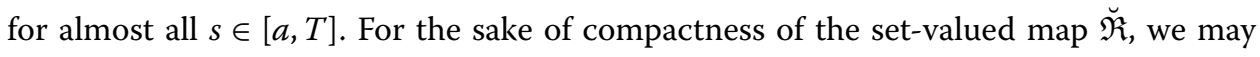
pass into a subsequence (if necessary) to obtain a convergent subsequence $\left\{\hat{\vartheta}_{n}\right\}_{n \geq 1}$ which 
converges to some $\hat{\vartheta} \in \mathcal{L}^{1}([a, T])$. Therefore, we have $\hat{\vartheta} \in(\mathfrak{S E} \mathfrak{L})_{\breve{\mathfrak{R}}, w}$, and so

$$
\begin{aligned}
\lim _{n \rightarrow \infty} w_{n}(s)= & \frac{1}{\Gamma(\varrho)} \int_{a}^{s}\left(\frac{(s-a)^{v}-(r-a)^{v}}{v}\right)^{\varrho-1} \hat{\vartheta}(r) \frac{\mathrm{d} r}{(r-a)^{1-v}} \\
& +\tilde{\Omega}_{1}(s) \frac{1}{\Gamma(\varrho)} \int_{a}^{T}\left(\frac{(T-a)^{v}-(r-a)^{v}}{v}\right)^{\varrho-1} \hat{\vartheta}(r) \frac{\mathrm{d} r}{(r-a)^{1-v}} \\
& +\tilde{\Omega}_{2}(s) \frac{1}{\Gamma\left(\varrho-p^{*}\right)} \int_{a}^{\xi}\left(\frac{(\xi-a)^{v}-(r-a)^{v}}{v}\right)^{\varrho-p^{*}-1} \hat{\vartheta}(r) \frac{\mathrm{d} r}{(r-a)^{1-v}} \\
& +\tilde{\Omega}_{3}(s) \frac{1}{\Gamma\left(\varrho+q^{*}\right)} \int_{a}^{\sigma}\left(\frac{(\sigma-a)^{v}-(r-a)^{v}}{v}\right)^{\varrho+q^{*}-1} \hat{\vartheta}(r) \frac{\mathrm{d} r}{(r-a)^{1-v}}+\tilde{\Omega}_{4}(s) \\
= & w(s)
\end{aligned}
$$

for each $s \in[a, T]$. From the above argument, we realize that $w \in \mathcal{K}(w)$ and thus $\mathcal{K}$ has closed values. By assumption of theorem, we know that $\breve{\mathfrak{R}}$ is a compact set-valued map, thus one can easily confirm that $\mathcal{K}(w)$ is a bounded set for each $w \in \mathcal{W}$. In this position, we are going to prove that the operator $\mathcal{K}$ is $\alpha$ - $\psi$-contractive. To observe that, formulate the nonnegative function $\alpha: \mathcal{W} \times \mathcal{W} \rightarrow[0, \infty)$ as $\alpha\left(w, w^{\prime}\right)=1$ if $\tilde{\zeta}\left(w(s), w^{\prime}(s)\right) \geq 0$ and $\alpha\left(w, w^{\prime}\right)=0$ otherwise. We also assume that $w, w^{\prime} \in \mathcal{W}$ and $z_{1} \in \mathcal{K}\left(w^{\prime}\right)$ are arbitrary. Select $\hat{\vartheta}_{1} \in(\mathfrak{S E L})_{\breve{R}, w^{\prime}}$ so that

$$
\begin{aligned}
z_{1}(s)= & \frac{1}{\Gamma(\varrho)} \int_{a}^{s}\left(\frac{(s-a)^{v}-(r-a)^{v}}{v}\right)^{\varrho-1} \hat{\vartheta}_{1}(r) \frac{\mathrm{d} r}{(r-a)^{1-v}} \\
& +\tilde{\Omega}_{1}(s) \frac{1}{\Gamma(\varrho)} \int_{a}^{T}\left(\frac{(T-a)^{v}-(r-a)^{v}}{v}\right)^{\varrho-1} \hat{\vartheta}_{1}(r) \frac{\mathrm{d} r}{(r-a)^{1-v}} \\
& +\tilde{\Omega}_{2}(s) \frac{1}{\Gamma\left(\varrho-p^{*}\right)} \int_{a}^{\xi}\left(\frac{(\xi-a)^{v}-(r-a)^{v}}{v}\right)^{\varrho-p^{*}-1} \hat{\vartheta}_{1}(r) \frac{\mathrm{d} r}{(r-a)^{1-v}} \\
& +\tilde{\Omega}_{3}(s) \frac{1}{\Gamma\left(\varrho+q^{*}\right)} \\
& \times \int_{a}^{\sigma}\left(\frac{(\sigma-a)^{v}-(r-a)^{v}}{v}\right)^{\varrho+q^{*}-1} \hat{\vartheta}_{1}(r) \frac{\mathrm{d} r}{(r-a)^{1-v}}+\tilde{\Omega}_{4}(s)
\end{aligned}
$$

for any $s \in[a, T]$. Condition (13) verifies that

$$
\operatorname{PH}_{d \mathcal{W}}\left(\breve{\mathfrak{R}}(s, w(s)), \breve{\mathfrak{R}}\left(s, w^{\prime}(s)\right)\right) \leq \breve{a}(s) \psi\left(\left|w(s)-w^{\prime}(s)\right|\right) \frac{1}{M\|\breve{a}\|}
$$

for all $w, w^{\prime} \in \mathcal{W}$ having the property $\tilde{\zeta}\left(w(s), w^{\prime}(s)\right) \geq 0$ for any $s \in[a, T]$. Hence, there exists $h \in \breve{\mathfrak{R}}(s, w(s))$ such that

$$
\left|\hat{\vartheta}_{1}(s)-h\right| \leq \breve{a}(s) \psi\left(\left|w(s)-w^{\prime}(s)\right|\right) \frac{1}{M\|\breve{a}\|} .
$$

In what follows, we build a new set-valued map $\mathcal{B}^{*}:[a, T] \rightarrow \mathcal{P}(\mathcal{W})$ as follows:

$$
\mathcal{B}^{*}(s)=\left\{h \in \mathcal{W}:\left|\hat{\vartheta}_{1}(s)-h\right| \leq \breve{a}(s) \psi\left(\left|w(s)-w^{\prime}(s)\right|\right) \frac{1}{M\|\breve{a}\|}\right\}
$$


for each $s \in[a, T]$. Because $\hat{\vartheta}_{1}$ and $\varpi=\breve{a} \psi\left(\left|w-w^{\prime}\right|\right) \frac{1}{M\|\breve{a}\|}$ are measurable, so the intersection of two set-valued maps $\mathcal{B}^{*}(\cdot) \cap \breve{\Re}(\cdot, w(\cdot))$ is measurable. For continuing the deduction, we choose $\hat{\vartheta}_{2}$ belonging to $\breve{\mathfrak{R}}(s, w(s))$ provided that

$$
\left|\hat{\vartheta}_{1}(s)-\hat{\vartheta}_{2}(s)\right| \leq \breve{a}(s) \psi\left(\left|w(s)-w^{\prime}(s)\right|\right) \frac{1}{M\|\breve{a}\|}
$$

for all $s \in[a, T]$. Define the element $z_{2} \in \mathcal{K}(w)$ by

$$
\begin{aligned}
z_{2}(s)= & \frac{1}{\Gamma(\varrho)} \int_{a}^{s}\left(\frac{(s-a)^{v}-(r-a)^{v}}{v}\right)^{\varrho-1} \hat{\vartheta}_{2}(r) \frac{\mathrm{d} r}{(r-a)^{1-v}} \\
& +\tilde{\Omega}_{1}(s) \frac{1}{\Gamma(\varrho)} \int_{a}^{T}\left(\frac{(T-a)^{v}-(r-a)^{v}}{v}\right)^{\varrho-1} \hat{\vartheta}_{2}(r) \frac{\mathrm{d} r}{(r-a)^{1-v}} \\
& +\tilde{\Omega}_{2}(s) \frac{1}{\Gamma\left(\varrho-p^{*}\right)} \int_{a}^{\xi}\left(\frac{(\xi-a)^{v}-(r-a)^{v}}{v}\right)^{\varrho-p^{*}-1} \hat{\vartheta}_{2}(r) \frac{\mathrm{d} r}{(r-a)^{1-v}} \\
& +\tilde{\Omega}_{3}(s) \frac{1}{\Gamma\left(\varrho+q^{*}\right)} \\
& \times \int_{a}^{\sigma}\left(\frac{(\sigma-a)^{v}-(r-a)^{v}}{v}\right)^{\varrho+q^{*}-1} \hat{\vartheta}_{2}(r) \frac{\mathrm{d} r}{(r-a)^{1-v}}+\tilde{\Omega}_{4}(s)
\end{aligned}
$$

for any $s \in[a, T]$. Then, one can compute the following estimate:

$$
\begin{aligned}
\mid z_{1}(s) & -z_{2}(s) \mid \\
\leq & \frac{1}{\Gamma(\varrho)} \int_{a}^{s}\left(\frac{(s-a)^{v}-(r-a)^{v}}{v}\right)^{\varrho-1}\left|\hat{\vartheta}_{1}(r)-\hat{\vartheta}_{2}(r)\right| \frac{\mathrm{d} r}{(r-a)^{1-v}} \\
& +\left|\tilde{\Omega}_{1}(s)\right| \frac{1}{\Gamma(\varrho)} \int_{a}^{T}\left(\frac{(T-a)^{v}-(r-a)^{v}}{v}\right)^{\varrho-1}\left|\hat{\vartheta}_{1}(r)-\hat{\vartheta}_{2}(r)\right| \frac{\mathrm{d} r}{(r-a)^{1-v}} \\
& +\left|\tilde{\Omega}_{2}(s)\right| \frac{1}{\Gamma\left(\varrho-p^{*}\right)} \\
& \times \int_{a}^{\xi}\left(\frac{(\xi-a)^{v}-(r-a)^{v}}{v}\right)^{\varrho-p^{*}-1}\left|\hat{\vartheta}_{1}(r)-\hat{\vartheta}_{2}(r)\right| \frac{\mathrm{d} r}{(r-a)^{1-v}} \\
& +\left|\tilde{\Omega}_{3}(s)\right| \frac{1}{\Gamma\left(\varrho+q^{*}\right)} \\
& \times \int_{a}^{\sigma}\left(\frac{(\sigma-a)^{v}-(r-a)^{v}}{v}\right)^{\varrho+q^{*}-1}\left|\hat{\vartheta}_{1}(r)-\hat{\vartheta}_{2}(r)\right| \frac{\mathrm{d} r}{(r-a)^{1-v}} \\
\leq & {\left[\frac{(T-a)^{v \varrho}}{v^{\varrho} \Gamma(\varrho+1)}+\Omega_{1}^{*} \frac{(T-a)^{v}}{v^{\varrho} \Gamma(\varrho+1)}\right.} \\
& \left.+\Omega_{2}^{*} \frac{(\xi-a)^{v\left(\varrho-p^{*}\right)}}{v^{\varrho-p^{*}} \Gamma\left(\varrho-p^{*}+1\right)}+\Omega_{3}^{*} \frac{(\sigma-a)^{v\left(\varrho+q^{*}\right)}}{v^{\varrho+q^{*}} \Gamma\left(\varrho+q^{*}+1\right)}\right] \\
& \times\|\breve{a}\| \psi\left(\left\|w-w^{\prime}\right\|\right) \frac{1}{M\|\breve{a}\|}=M\|\breve{a}\| \psi\left(\left\|w-w^{\prime}\right\|\right) \frac{1}{M\|\breve{a}\|}=\psi\left(\left\|w-w^{\prime}\right\|\right)
\end{aligned}
$$

for all $s \in[a, T]$. Hence, we find that

$$
\left\|z_{1}-z_{2}\right\|=\sup _{s \in[a, T]}\left|z_{1}(s)-z_{2}(s)\right| \leq \psi\left(\left\|w-w^{\prime}\right\|\right)
$$


and so we get $\alpha\left(w, w^{\prime}\right) \mathrm{PH}_{d_{\mathcal{W}}}\left(\mathcal{K}(w), \mathcal{K}\left(w^{\prime}\right)\right) \leq \psi\left(\left\|w-w^{\prime}\right\|\right)$ for each $w, w^{\prime} \in \mathcal{W}$ showing that the set-valued map $\mathcal{K}$ is $\alpha-\psi$-contractive. Now, consider two elements $w \in \mathcal{W}$ and $w^{\prime} \in \mathcal{K}(w)$ with $\alpha\left(w, w^{\prime}\right) \geq 1$. In the light of the definition of $\tilde{\zeta}$, we have an inequality $\tilde{\zeta}\left(w(s), w^{\prime}(s)\right) \geq 0$, and so there is a function $h \in \mathcal{K}\left(w^{\prime}\right)$ such that $\tilde{\zeta}\left(w^{\prime}(s), h(s)\right) \geq 0$. Hence, $\alpha\left(w^{\prime}, h\right) \geq 1$ and this states that $\mathcal{K}$ is $\alpha$-admissible.

To finish the rest of the proof, we suppose that $w_{0} \in \mathcal{W}$ and $w^{\prime} \in \mathcal{K}\left(w_{0}\right)$ are such that $\tilde{\zeta}\left(w_{0}(s), w^{\prime}(s)\right) \geq 0$ for each $s$. Thus, we get $\alpha\left(w_{0}, w^{\prime}\right) \geq 1$. On the other hand, consider the sequence $\left\{w_{n}\right\}_{n \geq 1}$ of $\mathcal{W}$ with $w_{n} \rightarrow w$ and $\alpha\left(w_{n}, w_{n+1}\right) \geq 1$ for each $n$. Then we have $\tilde{\zeta}\left(w_{n}(s), w_{n+1}(s)\right) \geq 0$. Now, with the help of (Hp4), we find that there is a subsequence $\left\{w_{n_{l}}\right\}_{l \geq 1}$ of $\left\{w_{n}\right\}$ such that $\tilde{\zeta}\left(w_{n_{l}}(s), w(s)\right) \geq 0$ for each $s \in[a, T]$. Consequently, $\alpha\left(w_{n_{l}}, w\right) \geq 1$ for all $l \geq 1$, and so it is verified that $\mathcal{W}$ has the property $\left(C_{\alpha}\right)$. Ultimately, in the light of Theorem 4 , we realize that the set-valued map $\mathcal{K}$ has a fixed point which is as a solution for the four-point Caputo conformable inclusion BVP (1)-(2).

By continuing the current process, we obtain another existence criterion for the fourpoint Caputo conformable inclusion BVP (1)-(2) under new analytical conditions. In other words, we shall prove our desired existence result under a new concept due to Amini-Harandi [39]. In this way, we use the approximate endpoint property for $\mathcal{K}$ which is defined by (12).

Theorem 9 Let $\breve{\mathfrak{R}}:[a, T] \times \mathcal{W} \rightarrow \mathcal{P}_{\text {cmp }}(\mathcal{W})$ be a compact set-valued map. In addition, suppose that the following statements are valid:

(Hp7) The nondecreasing nonnegative function $\psi:[0, \infty) \rightarrow[0, \infty)$ has the upper semi-continuity property so that $\liminf _{s \rightarrow \infty}(s-\psi(s))>0$ and $\psi(s)<s$ for each $s>0$;

(Hp8) The compact bounded operator $\breve{\Re}:[a, T] \times \mathcal{W} \rightarrow \mathcal{P}_{\text {cmp }}(\mathcal{W})$ is integrable provided that $\breve{\mathfrak{R}}(\cdot, w):[a, T] \rightarrow \mathcal{P}_{\mathrm{cp}}(\mathcal{W})$ is measurable for each $w \in \mathcal{W}$;

(Hp9) There is a nonnegative function $\delta \in \mathcal{C}_{\mathbb{R} \geq 0}([a, T])$ such that

$$
\operatorname{PH}_{d_{\mathcal{W}}}\left(\breve{\mathfrak{R}}(s, w)-\breve{\mathfrak{R}}\left(s, w^{\prime}\right)\right) \leq \delta(s) \psi\left(\left|w-w^{\prime}\right|\right) \frac{1}{M\|\delta\|}
$$

for all $s \in[a, T]$ and $w, w^{\prime} \in \mathcal{W}$, where $\sup _{s \in[a, T]}|\delta(s)|=\|\delta\|$ and $M$ is defined by (11);

(Hp10) The operator $\mathcal{K}$ defined by (12) has the approximate endpoint property.

Then the four-point Caputo conformable inclusion BVP (1)-(2) has a solution on $[a, T]$.

Proof As a general goal, we shall show the existence of an endpoint for the set-valued map $\mathcal{K}: \mathcal{W} \rightarrow \mathcal{P}(\mathcal{W})$. In this direction, we need to prove that the set $\mathcal{K}(w)$ is closed for each $w \in \mathcal{W}$. If we pay attention to hypothesis $(\mathrm{Hp} 8)$, then for the sake of the measurability of the map $s \mapsto \breve{\mathfrak{R}}(s, w(s))$ and closeness of it for all $w \in \mathcal{W}$, we realize that $\breve{\mathfrak{R}}$ has a measurable selection, and so $(\mathfrak{S E L})_{\breve{\mathfrak{R}}, w} \neq \emptyset$ for each $w \in \mathcal{W}$. Therefore, similar to the proof of last Theorem, it is easy to check that $\mathcal{K}(w)$ is a closed subset of $\mathcal{W}$, and hence we omit it. Moreover, we know that the set $\mathcal{K}(w)$ is bounded for each $w \in \mathcal{W}$ with due attention to the compactness of $\breve{\mathfrak{R}}$. We conclude the proof by proving the inequality $\mathrm{PH}_{d_{\mathcal{W}}}\left(\mathcal{K}(w), \mathcal{K}\left(w^{\prime}\right)\right) \leq$ $\psi\left(\left\|w-w^{\prime}\right\|\right)$ for every two arbitrary members of $\mathcal{W}$. To see this, assume that $w, w^{\prime} \in \mathcal{W}$ 
and $z_{1} \in \mathcal{K}\left(w^{\prime}\right)$. Choose $\hat{\vartheta}_{1} \in(\mathfrak{S E L})_{\breve{\Re}, w^{\prime}}$ so that

$$
\begin{aligned}
z_{1}(s)= & \frac{1}{\Gamma(\varrho)} \int_{a}^{s}\left(\frac{(s-a)^{v}-(r-a)^{v}}{v}\right)^{\varrho-1} \hat{\vartheta}_{1}(r) \frac{\mathrm{d} r}{(r-a)^{1-v}} \\
& +\tilde{\Omega}_{1}(s) \frac{1}{\Gamma(\varrho)} \int_{a}^{T}\left(\frac{(T-a)^{v}-(r-a)^{v}}{v}\right)^{\varrho-1} \hat{\vartheta}_{1}(r) \frac{\mathrm{d} r}{(r-a)^{1-v}} \\
& +\tilde{\Omega}_{2}(s) \frac{1}{\Gamma\left(\varrho-p^{*}\right)} \int_{a}^{\xi}\left(\frac{(\xi-a)^{v}-(r-a)^{v}}{v}\right)^{\varrho-p^{*}-1} \hat{\vartheta}_{1}(r) \frac{\mathrm{d} r}{(r-a)^{1-v}} \\
& +\tilde{\Omega}_{3}(s) \frac{1}{\Gamma\left(\varrho+q^{*}\right)} \\
& \times \int_{a}^{\sigma}\left(\frac{(\sigma-a)^{v}-(r-a)^{v}}{v}\right)^{\varrho+q^{*}-1} \hat{\vartheta}_{1}(r) \frac{\mathrm{d} r}{(r-a)^{1-v}}+\tilde{\Omega}_{4}(s)
\end{aligned}
$$

for almost all $s \in[a, T]$. Since, by considering inequality (14) presented in hypothesis (Hp9), we have

$$
\operatorname{PH}_{d_{\mathcal{W}}}\left(\breve{\mathfrak{R}}(s, w(s)), \breve{\mathfrak{R}}\left(s, w^{\prime}(s)\right)\right) \leq \delta(s) \psi\left(\left|w(s)-w^{\prime}(s)\right|\right) \frac{1}{M\|\delta\|}
$$

for any $s \in[a, T]$, thus there is $h^{*} \in \breve{\mathfrak{R}}(s, w(s))$, for which we get

$$
\left|\hat{\vartheta}_{1}(s)-h^{*}\right| \leq \delta(s) \psi\left(\left|w(s)-w^{\prime}(s)\right|\right) \frac{1}{M\|\delta\|}
$$

for all $s \in[a, T]$. Now, we build a new set-valued map $\mathcal{Q}:[a, T] \rightarrow \mathcal{P}(\mathcal{W})$ which is introduced by

$$
\mathcal{Q}(s)=\left\{h^{*} \in \mathcal{W}:\left|\hat{\vartheta}_{1}(s)-h^{*}\right| \leq \delta(s) \psi\left(\left|w(s)-w^{\prime}(s)\right|\right) \frac{1}{M\|\delta\|}\right\} .
$$

We know that $\hat{\vartheta}_{1}$ and $\varpi=\delta \psi\left(\left|w-w^{\prime}\right|\right) \frac{1}{M\|\delta\|}$ are measurable, so we can easily deduce that the intersection set-valued map $\mathcal{Q}(\cdot) \cap \breve{\mathfrak{R}}(\cdot, w(\cdot))$ is measurable. Now, we choose the function $\hat{\vartheta}_{2}(s) \in \breve{\mathfrak{R}}(s, w(s))$ so that

$$
\left|\hat{\vartheta}_{1}(s)-\hat{\vartheta}_{2}(s)\right| \leq \delta(s) \psi\left(\left|w(s)-w^{\prime}(s)\right|\right) \frac{1}{M\|\delta\|}
$$

for any $s \in[a, T]$. We also select $z_{2} \in \mathcal{K}(w)$ provided that

$$
\begin{aligned}
z_{2}(s)= & \frac{1}{\Gamma(\varrho)} \int_{a}^{s}\left(\frac{(s-a)^{v}-(r-a)^{v}}{v}\right)^{\varrho-1} \hat{\vartheta}_{2}(r) \frac{\mathrm{d} r}{(r-a)^{1-v}} \\
& +\tilde{\Omega}_{1}(s) \frac{1}{\Gamma(\varrho)} \int_{a}^{T}\left(\frac{(T-a)^{v}-(r-a)^{v}}{v}\right)^{\varrho-1} \hat{\vartheta}_{2}(r) \frac{\mathrm{d} r}{(r-a)^{1-v}} \\
& +\tilde{\Omega}_{2}(s) \frac{1}{\Gamma\left(\varrho-p^{*}\right)} \int_{a}^{\xi}\left(\frac{(\xi-a)^{v}-(r-a)^{v}}{v}\right)^{\varrho-p^{*}-1} \hat{\vartheta}_{2}(r) \frac{\mathrm{d} r}{(r-a)^{1-v}}
\end{aligned}
$$




$$
\begin{aligned}
& +\tilde{\Omega}_{3}(s) \frac{1}{\Gamma\left(\varrho+q^{*}\right)} \\
& \times \int_{a}^{\sigma}\left(\frac{(\sigma-a)^{v}-(r-a)^{v}}{v}\right)^{\varrho+q^{*}-1} \hat{\vartheta}_{2}(r) \frac{\mathrm{d} r}{(r-a)^{1-v}}+\tilde{\Omega}_{4}(s)
\end{aligned}
$$

for all $s \in[a, T]$. Therefore, by repeating a similar process in the proof of Theorem 8 , we obtain the following relations:

$$
\left\|z_{1}-z_{2}\right\|=\sup _{s \in[a, T]}\left|z_{1}(s)-z_{2}(s)\right| \leq M\|\delta\| \psi\left(\left\|w-w^{\prime}\right\|\right) \frac{1}{M\|\delta\|}=\psi\left(\left\|w-w^{\prime}\right\|\right) .
$$

This yields the inequality $\mathrm{PH}_{d_{\mathcal{W}}}\left(\mathcal{K}(w), \mathcal{K}\left(w^{\prime}\right)\right) \leq \psi\left(\left\|w-w^{\prime}\right\|\right)$ for each $w, w^{\prime} \in \mathcal{W}$. Furthermore, hypothesis (Hp10) confirms that $\mathcal{K}$ has the approximate endpoint property, so with due attention to Theorem 5 , we arrive at the desired conclusion which involves this property that the operator $\mathcal{K}$ has a unique endpoint, i.e., there is $w^{*} \in \mathcal{W}$ so that $\mathcal{K}\left(w^{*}\right)=\left\{w^{*}\right\}$. Thus, $w^{*}$ is a solution for the four-point Caputo conformable inclusion problem (1)-(2).

Now, we provide an example to illustrate Theorem 9.

Example 1 By considering the values $\varrho=1.5, v=1, p^{*}=0.04, q^{*}=0.2, \xi=\sigma=0.03$, $\mu_{1}=0.34, \mu_{2}=0.41, a=0, T=1$ and also according to problem (1)-(2), we formulate the Caputo fractional conformable differential inclusion

$$
{ }^{\mathrm{CC}} \mathcal{D}_{0}^{1,1.5} w(s) \in\left[0, \frac{0.75 s^{2}|\arcsin w(s)|}{|\arcsin w(s)|+1}\right] \quad(s \in[0,1])
$$

furnished with four-point mixed Riemann-Liouville conformable integro-derivative boundary conditions

$$
w(0)={ }^{\mathrm{RC}} \mathcal{D}_{0}^{1,0.04} w(0.03)+0.34, \quad w(1)={ }^{\mathrm{RC}} \mathcal{I}_{0}^{1,0.2} w(0.03)+0.41,
$$

so that ${ }^{\mathrm{CC}} \mathcal{D}_{0}^{1,1.5}$ is the Caputo conformable derivative of order $\varrho=1.5,{ }^{\mathrm{RC}} \mathcal{D}_{0}^{1,0.04}$ is the Riemann-Liouville conformable derivative of order $p^{*}=0.04$, and ${ }^{\mathrm{RC}} \mathcal{I}_{0}^{1,0.2}$ is the Riemann-Liouville conformable integral of order $q^{*}=0.2$. Due to the values mentioned above, we obtain $k_{0} \simeq-0.1227, k_{1} \simeq 0.03508, k_{2} \simeq-0.45986, k_{3} \simeq-0.98656$, and so $k=k_{0} k_{3}+k_{1} k_{2} \simeq 0.10492 \neq 0$. Prior to investigating the properties of desired set-valued map, we first define the Banach space $\mathcal{W}=\left\{w(s): w(s) \in \mathcal{C}_{\mathbb{R}}([0,1])\right\}$ furnished with the supremum norm $\|w\|_{\mathcal{W}}=\sup _{s \in[0,1]}|w(s)|$. Now, it is suitable to consider the set-valued $\operatorname{map} \breve{\mathfrak{R}}:[0,1] \times \mathcal{W} \rightarrow \mathcal{P}(\mathcal{W})$ by

$$
\breve{\mathfrak{R}}(s, w(s))=\left[0, \frac{0.75 s^{2}|\arcsin w(s)|}{|\arcsin w(s)|+1}\right]
$$

for any $s \in[0,1]$. According to the procedure mentioned in the proof of Theorem 9, we introduce the operator $\mathcal{K}: \mathcal{W} \rightarrow \mathcal{P}(\mathcal{W})$ given by

$$
\mathcal{K}(w)=\left\{z \in \mathcal{W} \text { : there is } \hat{\vartheta} \in(\mathfrak{S E E L})_{\breve{\mathfrak{R}}, w} \text { such that } z(s)=h(s) \text { for any } s \in[0,1]\right\},
$$


so that

$$
\begin{aligned}
h(s)= & \frac{1}{\Gamma(1.5)} \int_{0}^{s}\left(\frac{s^{1}-r^{1}}{1}\right)^{1.5-1} \hat{\vartheta}(r) \frac{\mathrm{d} r}{r^{1-1}} \\
& +\tilde{\Omega}_{1}(s) \frac{1}{\Gamma(1.5)} \int_{0}^{1}\left(\frac{1^{1}-r^{1}}{1}\right)^{1.5-1} \hat{\vartheta}(r) \frac{\mathrm{d} r}{r^{1-1}} \\
& +\tilde{\Omega}_{2}(s) \frac{1}{\Gamma(1.5-0.04)} \int_{0}^{0.03}\left(\frac{0.03^{1}-r^{1}}{1}\right)^{1.5-0.04-1} \hat{\vartheta}(r) \frac{\mathrm{d} r}{r^{1-1}} \\
& +\tilde{\Omega}_{3}(s) \frac{1}{\Gamma(1.5+0.2)} \int_{0}^{0.03}\left(\frac{0.03^{1}-r^{1}}{1}\right)^{1.5+0.2-1} \hat{\vartheta}(r) \frac{\mathrm{d} r}{r^{1-1}}+\tilde{\Omega}_{4}(s) \\
= & \frac{1}{\Gamma(1.5)} \int_{0}^{s}(s-r)^{1.5-1} \hat{\vartheta}(r) \mathrm{d} r+\tilde{\Omega}_{1}(s) \frac{1}{\Gamma(1.5)} \int_{0}^{1}(1-r)^{1.5-1} \hat{\vartheta}(r) \mathrm{d} r \\
& +\tilde{\Omega}_{2}(s) \frac{1}{\Gamma(1.5-0.04)} \int_{0}^{0.03}(0.03-r)^{1.5-0.04-1} \hat{\vartheta}(r) \mathrm{d} r \\
& +\tilde{\Omega}_{3}(s) \frac{1}{\Gamma(1.5+0.2)} \int_{0}^{0.03}(0.03-r)^{1.5+0.2-1} \hat{\vartheta}(r) \mathrm{d} r+\tilde{\Omega}_{4}(s),
\end{aligned}
$$

where we have the following estimates:

$$
\begin{aligned}
& \left|\tilde{\Omega}_{1}(s)\right|=|0.3343-1.1694 s| \leq|0.3343|+|1.1694 s| \leq \Omega_{1}^{*}:=1.5037 \\
& \left|\tilde{\Omega}_{2}(s)\right|=|-9.4029+4.3829 s| \leq|-9.4029|+|4.3829 s| \leq \Omega_{2}^{*}:=13.7858 \\
& \left|\tilde{\Omega}_{3}(s)\right|=|0.3343-1.1694 s| \leq|0.3343|+|1.1694 s| \leq \Omega_{3}^{*}:=1.5037 \\
& \left|\tilde{\Omega}_{4}(s)\right|=|-3.3330+1.9691 s| \leq|-3.3330|+|1.9691 s| \leq \Omega_{4}^{*}:=5.3021
\end{aligned}
$$

for each $s \in[0,1]$. Clearly, we have $M \simeq 1.952$. Moreover, the nondecreasing nonnegative function $\psi:[0, \infty) \rightarrow[0, \infty)$ with the upper semi-continuity property is considered as $\psi(s)=\frac{s}{2}$ for any $s>0$. Evidently, we have $\liminf _{s \rightarrow \infty}(s-\psi(s))>0$ and $\psi(s)<s$ for each $s>0$. In addition, for every $w, w^{\prime} \in \mathcal{W}$, we obtain the following inequality:

$$
\begin{aligned}
\operatorname{PH}_{d_{\mathcal{W}}}\left(\breve{\mathfrak{R}}(s, w(s)), \breve{\mathfrak{R}}\left(s, w^{\prime}(s)\right)\right) & \leq \frac{0.75 s^{2}}{2}\left(\left|w-w^{\prime}\right|\right) \\
& =0.75 s^{2} \psi\left(\left|w-w^{\prime}\right|\right) \leq \delta(s) \psi\left(\left|w-w^{\prime}\right|\right) \frac{1}{M\|\delta\|} .
\end{aligned}
$$

Letting $\delta \in \mathcal{C}_{\mathbb{R} \geq 0}([0,1])$ defined by $\delta(s)=0.75 s^{2}$ for each $s$, we have $\|\delta\|=0.75$, where we get $\frac{1}{M\|\delta\|} \simeq 0.68306$. Consequently, with the help of Theorem 9 on this numerical example, it is realized that the Caputo fractional conformable inclusion (15) with four-point mixed Riemann-Liouville conformable integro-derivative boundary conditions (16) has a solution, and thus the numerical findings of this example are consistent with the theoretical results presented in Theorem 9.

\section{Conclusion}

Our main goal in the present manuscript is to obtain some existence criteria for a new category of the Caputo conformable differential inclusion furnished with four-point mixed 
Riemann-Liouville conformable integro-derivative boundary conditions. Note that unlike other published papers in the field of existence theory, this inclusion problem supplemented with newly defined Caputo and Riemann-Liouville conformable operators is unique and this type of mixed inclusion problems has not been investigated in any literature. In this way, we utilize two concepts of fixed point and endpoint to obtain the existence criteria corresponding to the given BVP by (1)-(2). Indeed, some analytical techniques on the $\alpha-\psi$-contractive mappings and operators having the approximate endpoint property are employed to reach the desired theoretical results. Also, we provide an example to illustrate our last main result.

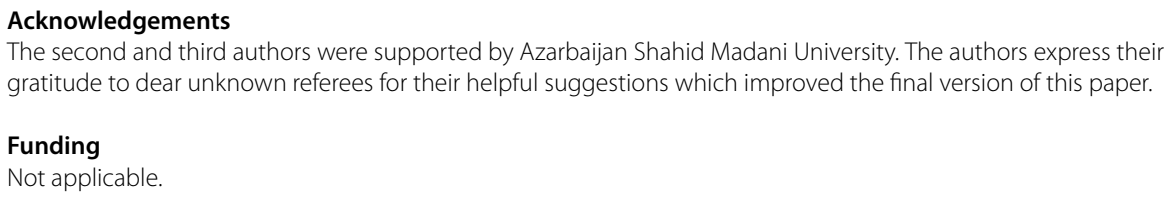

\section{Author details}

${ }^{1}$ Department of Mathematics, Cankaya University, Ogretmenler Cad. 14 06530, Balgat, Ankara, Turkey. ${ }^{2}$ Institute of Space Sciences, Magurele, Bucharest, Romania. ${ }^{3}$ Department of Mathematics, Azarbaijan Shahid Madani University, Tabriz, Iran. ${ }^{4}$ Institute of Research and Development, Duy Tan University, Da Nang 550000, Vietnam. ${ }^{5}$ Faculty of Natural Sciences, Duy Tan University, Da Nang 550000, Vietnam. ${ }^{6}$ Department of Medical Research, China Medical University Hospital, China Medical University, Taichung, Taiwan.

\section{Publisher's Note}

Springer Nature remains neutral with regard to jurisdictional claims in published maps and institutional affiliations.

Received: 29 May 2020 Accepted: 31 August 2020 Published online: 05 September 2020

\section{References}

1. Alsaedi, A., Ntouyas, S.K., Agarwal, R.P., Ahmad, B.: A nonlocal multi-point multi-term fractional boundary value problem with Riemann-Liouville type integral boundary conditions involving two indices. Adv. Differ. Equ. 2013, 369 (2013). https://doi.org/10.1186/1687-1847-2013-369

2. Baleanu, D., Etemad, S., Pourrazi, S., Rezapour, S.: On the new fractional hybrid boundary value problems with three-point integral hybrid conditions. Adv. Differ. Equ. 2019, 473 (2019)

3. Baleanu, D., Etemad, S., Rezapour, S.: A hybrid Caputo fractional modeling for thermostat with hybrid boundary value conditions. Bound. Value Probl. 2020, 64 (2020). https://doi.org/10.1186/s13661-020-01361-0

4. Li, B., Sun, S., Li, Y., Zhao, P.: Multi-point boundary value problems for a class of Riemann-Liouville fractional differential equations. Adv. Differ. Equ. 2014, 151 (2014). https://doi.org/10.1186/1687-1847-2014-151

5. Ntouyas, S.K., Etemad, S.: On the existence of solutions for fractional differential inclusions with sum and integral boundary conditions. Appl. Math. Comput. 266, 235-243 (2015). https://doi.org/10.1016/j.amc.2015.05.036

6. Benchohra, M., Lazreg, J.E.: Existence and Ulam stability for non-linear implicit fractional differential equations with Hadamard derivative. Stud. Univ. Babeş-Bolyai, Math. 62(1), 27-38 (2017)

7. Ntouyas, S.K., Tariboon, J.: Fractional integral problems for Hadamard-Caputo fractional Langevin differential inclusions. J. Appl. Math. Comput. 51, 13-33 (2016). https://doi.org/10.1007/s12190-015-0888-4

8. Tariboon, J., Ntouyas, S.K., Sudsutad, W.: Nonlocal Hadamard fractional integral conditions for nonlinear Riemann-Liouville fractional differential equations. Bound. Value Probl. 2014, 253 (2014). https://doi.org/10.1186/s13661-014-0253-9 
9. Wang, G., Pei, K., Agarwal, R.P., Zhang, L., Ahmad, B.: On the existence of solutions for fractional differential inclusions with sum and integral boundary conditions. J. Comput. Appl. Math. 343, 230-239 (2018). https://doi.org/10.1016/j.cam.2018.04.062

10. Caputo, M., Fabrizio, M.: A new definition of fractional derivative without singular kernel. Prog. Fract. Differ. Appl. 1(2), 73-85 (2015)

11. Losada, J., Nieto, J.J.: Properties of the new fractional derivative without singular kernel. Prog. Fract. Differ. Appl. 1(2), 87-92 (2015)

12. Aydogan, M., Baleanu, D., Mousalou, A., Rezapour, S.: On high order fractional integro-differential equations including the Caputo-Fabrizio derivative. Bound. Value Probl. 2018, 90 (2018). https://doi.org/10.1186/s13661-018-1008-9

13. Baleanu, D., Jajarmi, A., Mohammadi, H., Rezapour, S.: Analysis of the human liver model with Caputo-Fabrizio fractional derivative. Chaos Solitons Fractals 134, 7 (2020)

14. Baleanu, D., Rezapour, S., Saberpour, Z:: On fractional integro-differential inclusions via the extended fractional Caputo-Fabrizio derivation. Bound. Value Probl. 2019, 79 (2019). https://doi.org/10.1186/s13661-019-1194-0

15. Abdeljawad, T.: On conformable fractional calculus. J. Comput. Appl. Math. 279, 57-66 (2015). https://doi.org/10.1016/j.cam.2014.10.016

16. Khalil, R., Horani, M.A., Yousef, A., Sababheh, M.: A new definition of fractional derivative. J. Comput. Appl. Math. 264, 65-70 (2014). https://doi.org/10.1016/j.cam.2014.01.002

17. Jarad, F., Ugurlu, E., Abdeljawad, T., Baleanu, D.: On a new class of fractional operators. Adv. Differ. Equ. 2017, 247 (2017). https://doi.org/10.1186/s13662-017-1306-z

18. Katugampola, U.N.: A new approach to generalized fractional derivatives. Bull. Math. Anal. Appl. 6(4), 1-15 (2014)

19. Aphithana, A., Ntouyas, S.K., Tariboon, J.: Existence and Ulam-Hyers stability for Caputo conformable differential equations with four-point integral conditions. Adv. Differ. Equ. 2019, 139 (2019). https://doi.org/10.1186/s13662-019-2077-5

20. Baleanu, D., Mohammadi, H., Rezapour, S.: Analysis of the model of HIV-1 infection of CD4+ T-cell with a new approach of fractional derivative. Adv. Differ. Equ. 2020, 71 (2020). https://doi.org/10.1186/s13662-020-02544-W

21. Baleanu, D., Mohammadi, H., Rezapour, S.: A mathematical theoretical study of a particular system of Caputo-Fabrizio fractional differential equations for the rubella disease model. Adv. Differ. Equ. 2020, 184 (2020). https://doi.org/10.1186/s13662-020-02614-Z

22. Zhou, H., Alzabut, J., Rezapour, S., Samei, M.E.: Uniform persistence and almost periodic solutions of a non-autonomous patch occupancy model. Adv. Differ. Equ. 2020, 143 (2020) https://doi.org/10.1186/s13662-020-02603-2

23. Baleanu, D., Mohammadi, H., Rezapour, S.: A fractional differential equation model for the COVID-19 transmission by using the Caputo-Fabrizio derivative. Adv. Differ. Equ. 2020, 299 (2020). https://doi.org/10.1186/s13662-020-02762-2

24. Charandabi, Z.Z., Rezapour, S., Ettefagh, M.: On a fractional hybrid version of the Sturm-Liouville equation. Adv. Differ Equ. 2020, 301 (2020). https://doi.org/10.1186/s13662-020-02765-z

25. Etemad, S., Rezapour, S., Samei, M.E.: $\alpha$ - $\psi$-contractions and solutions of a $q$-fractional differential inclusion with three-point boundary value conditions via computational results. Adv. Differ. Equ. 2020, 218 (2020). https://doi.org/10.1186/s13662-020-02679-w

26. Etemad, S., Rezapour, S., Sakar, F.M.: On a fractional Caputo-Hadamard problem with boundary value conditions via different orders of the Hadamard fractional operators. Adv. Differ. Equ. 2020, 272 (2020). https://doi.org/10.1186/s13662-020-02741-7

27. Etemad, S., Rezapour, S.: On the existence of solutions for fractional boundary value problems on the Ethane graph. Adv. Differ. Equ. 2020, 276 (2020). https://doi.org/10.1186/s13662-020-02736-4

28. Amara, A., Etemad, S., Rezapour, S.: Topological degree theory and Caputo-Hadamard fractional boundary value problems. Adv. Differ. Equ. 2020, 369 (2020). https://doi.org/10.1186/s13662-020-02833-4

29. Baleanu, D., Ghafarnezhad, K., Rezapour, S., Shabibi, M.: On a strong-singular fractional differential equation. Adv. Differ. Equ. 2020, 350 (2020). https://doi.org/10.1186/s13662-020-02813-8

30. Aydogan, M., Baleanu, D., Mohammadi, H., Rezapour, S.: On the mathematical model of Rabies by using the fractional Caputo-Fabrizio derivative. Adv. Differ. Equ. 2020, 382 (2020). https://doi.org/10.1186/s13662-020-02798-4

31. Samei, M.E., Rezapour, S.: On a system of fractional $q$-differential inclusions via sum of two multi-term functions on a time scale. Bound. Value Probl. 2020, 135 (2020). https://doi.org/10.1186/s13661-020-01433-1

32. Tuan, N.H., Mohammadi, H., Rezapour, S.: A mathematical model for COVID-19 transmission by using the Caputo fractional derivative. Chaos Solitons Fractals 140, 110107 (2020). https://doi.org/10.1016/j.chaos.2020.110107

33. Podlubny, I.: Fractional Differential Equations. Academic Press, San Diego (1999)

34. Samko, S.G., Kilbas, A.A., Marichev, O.I.: Fractional Integrals and Derivatives: Theory and Applications. Gordon \& Breach, Philadelphia (1993)

35. Deimling, K.: Multi-Valued Differential Equations. de Gruyter, Berlin (1992)

36. Aubin, J., Cellina, A.: Differential Inclusions: Set-Valued Maps and Viability Theory. Springer, Berlin (1984) https://doi.org/10.1007/978-3-642-69512-4

37. Samet, B., Vetro, C., Vetro, P.: Fixed point theorems for $\alpha$ - $\psi$-contractive type mappings. Nonlinear Anal. 75 , 2154-2165 (2012)

38. Mohammadi, B., Rezapour, S., Shahzad, N.: Some results on fixed points of $\alpha$ - $\psi$-Ciric generalized multifunctions. Fixed Point Theory Appl. 2013, 24 (2013). https://doi.org/10.1186/1687-1812-2013-24

39. Amini-Harandi, A.: On Caputo type sequential fractional differential equations with nonlocal integral boundary conditions. Nonlinear Anal. 72, 132-134 (2010)

40. Lasota, A., Opial, Z.: An application of the Kakutani-Ky Fan theorem in the theory of ordinary differential equations. Bull. Acad. Pol. Sci., Sér. Sci. Math. Astron. Phys. 13, 781-786 (1965) 